\title{
Um Modelo para a Determinação do Equilíbrio Térmico de Bovinos em Ambientes Tropicais ${ }^{1}$
}

\author{
Roberto Gomes da Silva ${ }^{2}$
}

\begin{abstract}
RESUMO - Uma equação com base na teoria da transferência térmica foi apresentada para estimativa da termólise em bovinos expostos a um ambiente tropical especificado por temperatura e umidade do ar, radiação solar e vento. As variáveis do animal (taxa de sudação, temperatura retal, frequência respiratória, temperatura da superfície cutânea, coloração do pelame e peso corporal) foram consideradas em conjunto com as variáveis ambientais (temperatura do ar e umidade, radiação solar, velocidade do vento e temperatura do globo). A equação permite a predição de: (1) o nível de termogênese metabólica necessária para equilibrar as perdas térmicas sob condições ambientais especificadas; (2) a combinação de fatores ambientais que proporcionam determinado fluxo de calor entre um dado animal e o seu ambiente.
\end{abstract}

Palavras-chave: bovinos, modelo, termorregulação

\section{A Heat Balance Model for Cattle in Tropical Environments}

\begin{abstract}
An equation based on heat transfer theory was presented to estimate the rate of heat loss from cattle exposed to a tropical environment, specified by the air temperature, humidity, solar radiation, and wind speed. The animals' variables (sweating rate, rectal temperature, respiratory rate, surface temperature, haircoat color, and body weight) were considered together with environmental variables (air temperature and humidity, solar radiation, wind speed, and globe temperature). The equation allows the prediction of (a) the metabolic heat production level necessary to balance heat losses under specified environmental conditions; (b) the combination of environmental factors that provide a determined heat flux between a given animal and its environment.
\end{abstract}

Key Words: cattle, model, thermoregulation

\section{Introdução}

Existe crescente interesse em modelos que descrevem a termorregulação de animais domésticos, visando sobretudo a previsão ou estimação das reações dos mesmos em ambientes específicos. $\mathrm{O}$ ambiente térmico, principalmente em condições de campo, é bastante complexo, limitando sensivelmente a determinação da termorregulação, uma vez que a radiação, a velocidade do vento, a umidade e a temperatura do ar modificam-se no tempo e no espaço. Essas variáveis interagem entre si e com diversas características dos organismos, de modo que a alteração de uma única variável ambiental pode alterar consideravelmente todos os fatores envolvidos no equilíbrio térmico dos animais.

Numa região intertropical (entre os paralelos de $23^{\circ}$, norte e sul) a temperatura do ar encontra-se freqüentemente próxima da corporal ou a excede; além disso, a temperatura radiante média do ambiente tende a ser muito mais elevada que a atmosférica; conseqüentemente, a termólise por convecção e radiação é dificultada ou inibida. Em adição, se a região for também úmida, a perda de calor por evaporação será prejudicada, de modo que o estresse proporcionado sobre os animais pode ser muito elevado.

Tais condições são muito diferentes das que ocorrem nas regiões temperadas, nas quais a temperatura do ar é quase sempre bem mais baixa que a corporal e a temperatura radiante média raramente ultrapassa a temperatura do ar, além do fato de a irradiância solar de ondas curtas ser muito menor que aquela em latitudes mais baixas. Assim, os modelos estabelecidos para essas regiões podem não atender aos requisitos para as zonas intertropicais.

A literatura referente a modelos de equilíbrio térmico para animais, em geral, é limitada; para bovinos, em particular, podem-se citar apenas PORTER e GATES (1969), MONTEITH (1974) e McARTHUR (1987).

\footnotetext{
${ }^{1}$ Financiado pela Fundação de Amparo à Pesquisa do Estado de São Paulo, proc. FAPESP 97/09717-0.

2 Med.Vet., M.Sc., D.Sc., Livre Docente, Professor Titular de Bioclimatologia, Departamento de Zootecnia, Faculdade de Ciências Agrárias e Veterinárias, Universidade Estadual Paulista, Campus de Jaboticabal, 14870-000, Jaboticabal, SP. E-mail: rgomes@fcav.unesp.br
} 
No presente trabalho, procurou-se estabelecer um modelo abrangente e, ao mesmo tempo, tão simples quanto possível, para a determinação do equilíbrio térmico de bovinos em ambientes tropicais.

Aspectos teóricos

A energia térmica presente no organismo de um animal homeotérmico, tal como um bovino, é em sua maior parte gerada pelos processos metabólicos (M), mas uma porção significativa é procedente do meio ambiente, por meio de radiação de ondas curtas $\left(\mathbf{R}_{\mathbf{C}}\right)$ e longas $\left(\mathbf{R}_{\mathbf{L}}\right)$. Em adição, há uma quantidade de energia estocada (S). O afluxo por convecção e condução pode ser considerado desprezível, em virtude de: (a) ser pouco freqüente a temperatura ambiente exceder a temperatura corporal; (b) a temperatura da superfície ser quase sempre mais elevada que a da atmosfera; e (c) o contato físico direto entre as superfícies do corpo e as do ambiente ser bastante limitado, exceto no caso de animais deitados, sabendo-se que os animais em ambiente tropical dificilmente procuram contato com superfícies cuja temperatura seja maior que a corporal. Assim, a equação do equilíbrio térmico nos bovinos pode ser expressa como:

$$
\mathbf{M}+\mathbf{R}_{\mathbf{C}} \boldsymbol{\alpha}-\mathbf{S}=\mathbf{R}_{\mathbf{L}}+\mathbf{C}_{\mathbf{S}}+\mathbf{E}_{\mathbf{S}}+\mathbf{C}_{\mathbf{R}}+\mathbf{E}_{\mathbf{R}}
$$

em que $\boldsymbol{\alpha}$ é o coeficiente de absorvidade da superfície externa do corpo para a radiação de ondas curtas; $\mathbf{C}_{\mathbf{S}}$ e $\mathbf{C}_{\mathbf{R}}$, as perdas por convecção cutânea e respiratória, respectivamente; e $\mathbf{E}_{\mathbf{S}}$ e $\mathbf{E}_{\mathbf{R}}$, as perdas de calor latente por evaporação cutânea e respiratória, respectivamente.

\section{Aporte e estocagem de energia térmica}

Na relação [1], M representa a quantidade de energia gerada por um nível metabólico mínimo, sob condições de repouso e quando o ciclo de atividade orgânica se encontra em uma fase mais baixa (primeiras horas do dia), conforme definição de OWEN et al. (1986). Este valor pode ser denominado metabolismo de repouso, sendo adotado em lugar do metabolismo basal, devido ao fato de que as condições deste último só podem ser atendidas, a rigor, em seres humanos. Além disso, interessa-nos a termogênese ocorrida em condições normais da vida de um animal.

A termogênese, em geral, é estimada a partir do consumo de $\mathrm{O}_{2}$ e da eliminação de $\mathrm{CO}_{2}$, mas eventualmente isso pode não ser possível, sendo uma alternativa - quando não se requer precisão - o emprego da relação entre metabolismo mínimo e peso corporal (KLEIBER 1961).

Conforme SCHMIDT-NIELSEN (1989), quando se comparam entre si animais com diferentes pesos corporais, deve-se considerar a taxa metabólica específica, isto é, por unidade de massa corporal. Então, considerando a equação de KLEIBER (1961), tem-se:

$$
\mathbf{M}=\left(3,3922 \mathrm{P}^{0,75}\right) / \mathrm{P}=3,3922 \mathrm{P}^{-0,25}, \mathrm{~W} \cdot \mathrm{kg}^{-1} \quad \text { [2] }
$$

em que $\mathrm{P}$ é o peso corporal (kg). Nota-se que, quanto maior a massa corporal, menor é a produção de calor por unidade de peso. Entretanto, as trocas de energia térmica são expressas em termos de área de superfície, e não de massa, de modo que, para compatibilizar a expressão de $\mathbf{M}$ com a dos demais componentes de [1], o divisor na equação [2] deve ser a área que, para bovinos de raças européias, pode ser estimada por $\mathrm{A}=$ 0,13 $\mathrm{P}^{5 / 9}, \mathrm{~m}^{2}$ (KIBLER e BRODY, 1950; FINCH, 1985). Substituindo $P$ por A na equação [2], obtém-se:

$$
\mathbf{M}=26,093846 \mathrm{P}^{7 / 36}, \mathrm{~W} \cdot \mathrm{m}^{-2} \quad \text { [ 2a] }
$$

A estocagem de energia, $\mathbf{S}$, também se relaciona com a massa corporal, mas em forma positiva, havendo, portanto, concordância com a fórmula [2]. Em outras palavras, indivíduos mais pesados armazenam maior quantidade de energia e necessitam de taxa de produção de calor proporcionalmente menor.

De modo geral, a estocagem térmica pode ser medida como a diferença entre a perda de calor e a termogênese, durante dado intervalo de tempo:

$$
\mathbf{S}=\mathrm{c}_{\mathrm{pt}} \mathrm{P} d \mathrm{~T}
$$

em que $\mathrm{c}_{\mathrm{pt}}$ é o calor específico médio dos tecidos e $d \mathrm{~T}$, o diferencial de temperatura do corpo no intervalo de tempo (BLAXTER 1989). Muitos autores consideram o valor $\mathrm{c}_{\mathrm{pt}}=3470 \mathrm{~J} \cdot \mathrm{kg}^{-1} \cdot{ }^{\mathrm{o}} \mathrm{K}^{-1}$, porém estudos mais recentes têm indicado o valor $3340 \mathrm{~J} \cdot \mathrm{kg}^{-1} \cdot{ }^{\mathrm{o}} \mathrm{K}^{-1}$. Indivíduos muito gordos, com cerca de $50 \%$ de gordura corporal, apresentam $\mathrm{c}_{\mathrm{pt}}=2730 \mathrm{~J} \cdot \mathrm{kg}^{-1} \cdot{ }^{\mathrm{o}} \mathrm{K}^{-1}$. Uma vez que a temperatura corporal varia bastante conforme o local, uma temperatura média pode ser obtida como

$$
\overline{\mathrm{T}}_{\mathrm{B}}=\beta \mathrm{T}_{\mathrm{B}}+(1-\beta) \mathrm{T}_{\mathrm{s}}
$$

em que $\mathrm{T}_{\mathrm{B}}$ é a temperatura retal e TS, a temperatura da superfície cutânea, ambas em graus ${ }^{\circ} \mathrm{K}$. Daqui em diante, adotar-se-á a convenção de representar as temperaturas em "t" minúsculo para graus ${ }^{\circ} \mathrm{C}$ e em "T" maiúsculo para graus ${ }^{\circ} \mathrm{K}$.

McLEAN et al. (1983 a,b) determinaram a estocagem térmica em bovinos submetidos a temperaturas ambientais entre 12 e $25^{\circ} \mathrm{C}$, estabelecendo 
1246 Rev. bras. zootec.

um coeficiente $\beta=0,86$ e a equação

$$
\mathbf{S}=\mathrm{c}_{\mathrm{pt}}\left[0,86 \Delta \mathrm{T}_{\mathrm{B}}+0,14 \Delta \mathrm{T}_{\mathrm{S}}\right] / \mathrm{t}, \mathrm{W} \cdot \mathrm{kg}^{-1} \quad \text { [3] }
$$

em que $\Delta \mathrm{T}_{\mathrm{B}}$ e $\Delta \mathrm{T}_{\mathrm{S}}$ são os diferenciais da temperatura retal e da temperatura cutânea respectivamente e $t$, o período de tempo em segundos.

$\mathrm{O}$ valor $\mathbf{R}_{\mathrm{C}}$ representa a irradiância do corpo do animal por ondas curtas, podendo ser obtida pelas fórmulas descritas por SILVA (1999), o qual simbolizou esse valor como $\mathbf{S}_{\mathrm{t}}$. Se o animal estiver em um recinto fechado, esse termo é nulo; se estiver sob uma sombra no campo, apenas a radiação direta é eliminada, calculando-se a radiação difusa e a refletida. Quanto ao coeficiente de absorvidade da superfície do pêlame, $\alpha$, na Tabela 3 encontram-se alguns valores encontrados na literatura.

\section{Eliminação de calor}

As trocas de calor sensível na superfície corporal ocorrem por meio de convecção e de radiação de ondas longas,

$$
\mathbf{R}_{\mathbf{L}}+\mathbf{C}_{\mathrm{S}}=\varepsilon \sigma\left(\mathrm{T}_{\mathrm{S}}^{4}-\mathrm{T}_{\mathrm{RM}}^{4}\right)+h_{\mathrm{C}}\left(\mathrm{T}_{\mathrm{S}}-\mathrm{T}_{\mathrm{A}}\right)
$$

em que e é a emissividade; $\mathrm{T}_{S}$, a temperatura da superfície corporal; $\sigma$ é a constante de StefanBoltzmann (= 5,6697 x 10-8 W.m $\left.{ }^{-2} \cdot{ }^{\circ} \mathrm{K}^{-4}\right) ; \mathrm{T}_{\mathrm{RM}}$, a temperatura radiante média do ambiente; $\mathrm{T}_{\mathrm{A}}$, a temperatura do ar e $h_{\mathrm{C}}$, o coeficiente de convecção.

A compatibilização dos dois termos à direita da equação [4] e a linearidade da mesma requerem que ambos envolvam potências unitárias. Se uma potência unitária for usada na descrição das trocas por radiação, será constatado que os processos $\mathbf{R}_{\mathrm{L}}$ e $\mathbf{C}_{\mathrm{S}}$ têm em comum os fatos de dependerem da temperatura da superfície e de uma temperatura ambiental $\left(\mathrm{T}_{\mathrm{a}}\right.$ ou $\mathrm{T}_{\mathrm{RM}}$ ) e ainda de dois coeficientes. Calculando a derivada da função $\mathbf{R}_{\mathrm{L}}=\varepsilon \sigma\left(\mathrm{T}_{\mathrm{S}}^{4}-\mathrm{T}_{\mathrm{RM}}^{4}\right)$ com respeito a $\mathrm{T}_{\mathrm{RM}}$, obtém-se

$$
h_{\mathrm{R}}=\frac{d \mathbf{R}_{\mathrm{L}}}{d \mathrm{~T}_{\mathrm{RM}}}=4 \varepsilon \sigma \mathrm{T}_{\mathrm{RM}}^{3}
$$

e, assim, sendo $\mathrm{r}_{\mathrm{R}}=\mathrm{PC}_{\mathrm{p}} /\left(4 \varepsilon \sigma \mathrm{TaT}_{\mathrm{RM}}^{3}\right)$ a resistência à transferência de calor por radiação (em unidades s. $\mathrm{m}^{-1}$ ), vem

$$
\mathrm{RL}=h_{\mathrm{R}}\left(\mathrm{T}_{\mathrm{S}}-\mathrm{T}_{\mathrm{RM}}\right)=\frac{\rho c_{\mathrm{p}}\left(\mathrm{T}_{\mathrm{S}}-\mathrm{T}_{\mathrm{RM}}\right)}{\mathrm{r}_{\mathrm{R}}}
$$

em que $c_{p}$ é o calor específico do ar à temperatura $T_{A}$.

Pode ser facilmente demonstrado que o erro de estimação pela fórmula [6] em relação à equação original é pequeno, apenas $2,3 \%$, quando a diferença de temperatura $\left(\mathrm{T}_{\mathrm{S}}-\mathrm{T}_{\mathrm{RM}}\right)$ é de $5^{\circ} \mathrm{K}$ e $7,4 \%$, quando a diferença é de $15^{\circ} \mathrm{K}$.
Para se obter o coeficiente de convecção $h_{\mathrm{C}}$, considera-se o corpo de um bovino como constituindo um cilindro horizontal de diâmetro d (em metros), sendo

$$
h_{\mathrm{C}}=\mathrm{k} \mathrm{d}^{-1} \mathrm{~N}_{\mathrm{u}}
$$

em que $\mathrm{k}$ é a condutividade térmica do ar à temperatura $\mathrm{T}_{\mathrm{A}}$ e $\mathrm{N}_{\mathrm{u}}$, o número de Nusselt para um cilindro horizontal. O cálculo de $\mathrm{N}_{\mathrm{u}}$ requer três outros valores adimensionais:

número de Reynolds, $\mathrm{R}_{\mathrm{e}}=\mathrm{V} \mathrm{d} \mathrm{v}^{-1}$

número de Grashof, $\mathrm{G}_{\mathrm{r}}=\mathrm{gd}^{3} \mathrm{v}^{-2} \mathrm{~T}_{\mathrm{A}}^{-1}\left(\mathrm{~T}_{\mathrm{S}}-\mathrm{T}_{\mathrm{A}}\right)$

número de Prandtl, $\mathrm{P}_{\mathrm{r}}=\rho \mathrm{c}_{\mathrm{p}} v \mathrm{k}^{-1}$

em que $\mathrm{V}$ é a velocidade do vento $\left(\mathrm{m} . \mathrm{s}^{-1}\right) ; \mathrm{g}$, a aceleração da gravidade $\left(\mathrm{m} . \mathrm{s}^{-2}\right) ; \mathrm{v}, \rho$ e $\mathrm{c}_{\mathrm{p}}$, a viscosidade cinemática, a densidade e o calor específico do ar à temperatura $\mathrm{T}_{\mathrm{A}}$. No Apêndice, são dadas fórmulas para a estimação desses parâmetros.

Considerando a convecção forçada, conforme CHAPMAN (1987), o número de Nusselt é dado por:

$$
\begin{aligned}
\mathrm{N}_{\mathrm{u}} & =0,26 \mathrm{R}_{\mathrm{e}}^{0,6} \mathrm{P}_{\mathrm{r}}^{0,37}\left(\text { para } \mathrm{R}_{\mathrm{e}} \leq 2 \times 105\right) \\
& =0,076 \mathrm{R}_{\mathrm{e}}^{0,7} \mathrm{P}_{\mathrm{r}}^{0,37}\left(\text { para } \mathrm{R}_{\mathrm{e}}>2 \times 105\right)
\end{aligned}
$$

ao passo que, para convecção natural,

$$
\begin{aligned}
\mathrm{N}_{\mathrm{u}} & =0,52 \mathrm{G}_{\mathrm{r}}^{1 / 4} \operatorname{Pr}^{1 / 4}(\text { para } \mathrm{Gr} \leq 109) \\
& =0,098 \mathrm{G}_{\mathrm{r}}^{1 / 3} \operatorname{Pr}^{1 / 4}(\text { para } \mathrm{Gr}>109)
\end{aligned}
$$

de acordo com MONTEITH e UNSWORTH (1991).

Quando a velocidade do vento é muito baixa $\left(<1 \mathrm{~m} \cdot \mathrm{s}^{-1}\right)$, podem ocorrer simultaneamente ambas as formas de convecção. Neste caso, podese estabelecer a forma predominante calculando $x=\mathrm{G}_{\mathrm{r}} / \mathrm{R}_{\mathrm{e}}{ }^{2}$. Para CHAPMAN (1987), se $x \leq 0,08$, deve-se considerar a convecção forçada; se $x>3$, considera-se a convecção natural; e se $0,08<x<3$, determina-se $\mathrm{N}_{\mathrm{u}}$ para ambas as formas de convecção, escolhendo-se o maior valor.

A eliminação de calor latente pelos animais processa-se por evaporação cutânea $\left(\mathbf{E}_{\mathrm{S}}\right)$ e evaporação respiratória $\left(\mathbf{E}_{\mathrm{R}}\right)$,

$\mathbf{E}_{\mathrm{S}}+\mathbf{E}_{\mathrm{R}}=h_{\mathrm{Es}}\left(e_{\mathrm{S}}\left\{\mathrm{T}_{\mathrm{S}}\right\}-e\left\{\mathrm{~T}_{\mathrm{A}}\right\}\right)+h_{\mathrm{Er}}\left(e_{\mathrm{S}}\left\{\mathrm{T}_{\mathrm{B}}\right\}-e\left\{\mathrm{~T}_{\mathrm{A}}\right\}\right)$ [15]

em que $h_{\mathrm{Es}}$ e $h_{\mathrm{Er}}$ são os coeficientes de transferência de calor latente por evaporação cutânea e respiratória, respectivamente; $e_{S}\left\{\mathrm{~T}_{\mathrm{S}}\right\}$, a pressão de saturação de vapor do ar à temperatura $\mathrm{T}_{\mathrm{S}} ; e_{\mathrm{S}}\left\{\mathrm{T}_{\mathrm{B}}\right\}$, a pressão de saturação à temperatura no interior dos pulmões; e $e\left\{\mathrm{~T}_{\mathrm{A}}\right\}$, a pressão parcial de vapor na atmosfera à temperatura $\mathrm{T}_{\mathrm{A}}$.

Pela teoria corrente, o vapor é eliminado nas vias respiratórias à temperatura corporal profunda $\left(\mathrm{T}_{\mathrm{B}}\right)$, de modo que o ar estaria saturado a esta temperatura. Entretanto, observações experimentais de STEVENS 
(1981) demonstram que a eliminação respiratória de vapor em vacas é consideravelmente superestimada, quando se baseia na expiração de ar saturado à temperatura $\mathrm{T}_{\mathrm{B}}$. Isso ocorre porque as passagens aéreas (vias nasais, laringe, traquéia e grandes brônquios) apresentam superfícies resfriadas pela passagem do ar proveniente do exterior e por evaporação prévia da umidade, de modo que o ar expirado se encontra realmente a uma temperatura mais baixa, que pode ser estimada por:

$$
t_{R}=17+0,3 t_{A}+\exp \left\{0,01611 u_{r}+0,0387 t_{A}\right\},{ }^{\circ} \mathrm{C} \quad \text { [16] }
$$
em que $t_{\mathrm{A}}$ é a temperatura da atmosfera e $u_{r}$, a umidade relativa (\%). Segundo STEVENS (1981), a determinação do calor eliminado por evaporação respiratória, com base na temperatura $t_{R}$, apresenta resultados em concordância muito estreita com valores obtidos a partir de medições empíricas da quantidade de vapor exalado.

Os coeficientes de evaporação são dados pelas fórmulas:

$$
\begin{gathered}
h_{\mathrm{Es}}=\frac{\rho c_{\mathrm{p}} D \cdot S_{\mathrm{h}}}{\gamma \mathrm{d}}=\frac{\mathrm{p} \mathrm{c}_{\mathrm{p}}}{\gamma \mathrm{r}_{\mathrm{v}}} \\
h_{\mathrm{Er}}=\frac{\rho c_{\mathrm{p}}}{\gamma \mathrm{r}_{\mathrm{U}}}
\end{gathered}
$$

em que $\gamma$ é a constante psicrométrica; $D$, a difusividade do vapor de água à temperatura $\mathrm{T}_{\mathrm{A}}$; $\mathrm{r}_{\mathrm{V}}=\mathrm{d}\left(D \cdot \mathrm{S}_{\mathrm{h}}\right)^{-1}$, a resistência à transferência de vapor na superfície corporal $\left(\mathrm{s} \cdot \mathrm{m}^{-1}\right) ; \mathrm{e}_{\mathrm{h}}$, o número de Sherwood,

$$
\mathrm{S}_{\mathrm{h}}=\mathrm{N}_{\mathrm{u}}\left(\frac{v}{D \cdot \mathrm{P}_{\mathrm{r}}}\right)^{m}
$$

sendo $m$ a potência de $\mathrm{P}_{\mathrm{r}}(0,37$ ou 0,25$)$ na equação correspondente de $\mathrm{N}_{\mathrm{u}} ; \mathrm{r}_{\mathrm{U}}$, a resistência à transferência de vapor na respiração de vacas, conforme McARTHUR (1987),

$$
r_{u}=100 /(0,005+0,00027 F) \quad[20]
$$

em unidades s.m ${ }^{-1}$, em que $\mathrm{F}$ é a frequência respiratória por minuto.

\section{Material e Métodos}

\section{Desenvolvimento das equações}

Consideremos a Figura 1. Constata-se que ofluxo de calor sensível entre a epiderme e o ambiente é representado por

$$
\mathbf{R}_{\mathrm{L}}+\mathbf{C}_{\mathrm{S}}=\frac{\rho \mathrm{c}_{\mathrm{p}}\left(\mathrm{T}_{\mathrm{S}}-\mathrm{T}_{\mathrm{RM}}\right)}{\mathrm{r}_{\mathrm{R}}}+\frac{\rho \mathrm{c}_{\mathrm{p}}\left(\mathrm{T}_{\mathrm{S}}-\mathrm{T}_{\mathrm{A}}\right)}{\mathrm{r}_{\mathrm{C}}}
$$

em que $r_{c}=\rho c_{p} d /\left(k \cdot N_{u}\right)$. Todavia, há nesse conjunto duas diferentes temperaturas ambientais, $\mathrm{T}_{\mathrm{A}} \mathrm{e}$
$\mathrm{T}_{\mathrm{RM}}$, sendo conveniente reduzi-las a uma única. Aplicando o conceito da temperatura operativa (HERRINGTON et al. 1937) e lembrando que as resistências $r_{R}$ e $r_{C}$ se acham em paralelo entre si, de modo tal que a respectiva resistência equivalente é $r_{0}$ $=\mathrm{r}_{\mathrm{R}} \mathrm{r}_{\mathrm{C}} /\left(\mathrm{r}_{\mathrm{R}}+\mathrm{r}_{\mathrm{C}}\right)$, ter-se-á então:

$$
\frac{\rho c_{p}\left(T_{S}-T_{0}\right)}{r_{0}}=\frac{\rho c_{p}\left(T_{S}-T_{R M}\right)}{r_{R}}+\frac{\rho c_{p}\left(T_{S}-T_{A}\right)}{r_{C}}
$$

Resolvendo para $\mathrm{T}_{0}$, obtém-se a temperatura operativa:

$$
\mathrm{T}_{0}=\frac{\mathrm{r}_{\mathrm{C}} \mathrm{T}_{\mathrm{RM}}+\mathrm{r}_{\mathrm{R}} \mathrm{T}_{\mathrm{A}}}{\mathrm{r}_{\mathrm{R}}+\mathrm{r}_{\mathrm{C}}},{ }^{\circ} \mathrm{K}
$$

O fluxo de calor latente por evaporação na superfície cutânea é expresso por

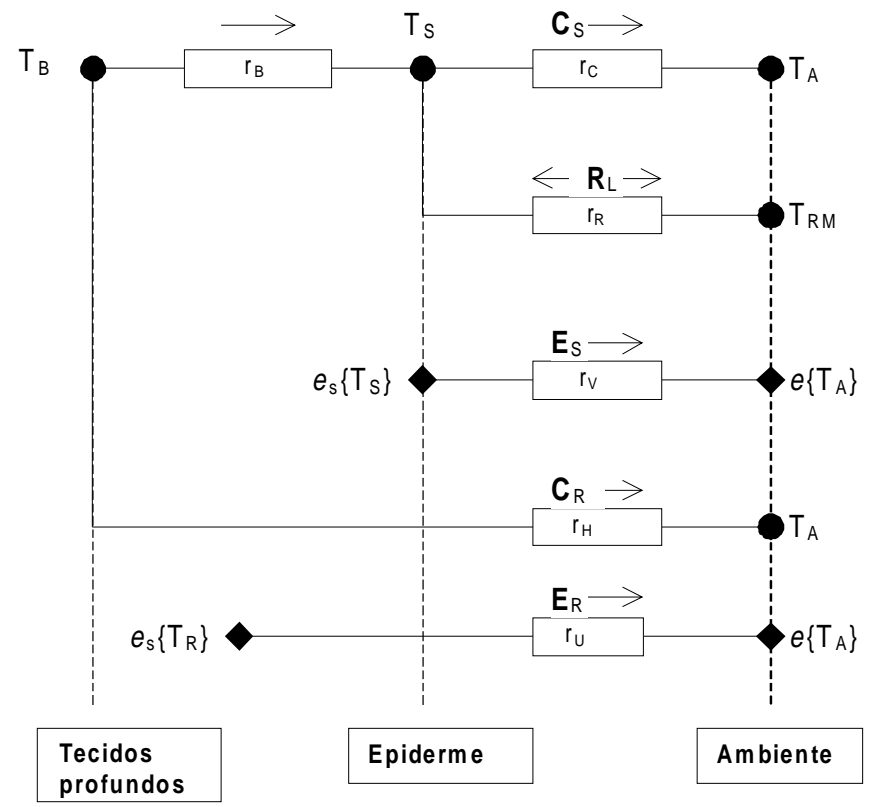

Figura 1 - Esquema do fluxo de energia térmica saindo do organismo de um bovino. $\mathrm{T}_{\mathrm{A}}=$ temperatura do ar, $\mathrm{T}_{\mathrm{RM}}=$ temperatura radiante média, $e\left\{\mathrm{~T}_{\mathrm{A}}\right\}=$ pressão parcial de vapor da atmosfera, $T_{S}=$ temperatura cutânea, $\mathrm{T}_{\mathrm{B}}=$ temperatura corporal profunda, $\mathbf{C}_{\mathrm{S}}=$ fluxo por convecção, $\mathbf{R}_{\mathrm{L}}=$ trocas por radiação de ondas longas, $\mathbf{E}_{S}=$ fluxo por evaporação cutânea, $\mathbf{C}_{R}=$ fluxo respiratório de calor sensível, $\mathbf{E}_{R}=$ fluxo por evaporação respiratória, $e_{S}\left\{T_{S}\right\}=$ pressão de saturação de vapor junto à epiderme, $e_{s}\left\{T_{R}\right\}=$ pressão de saturação de vapor do ar expirado à temperatura $T_{R}$.

Figure 1 - Heat flux from the body of cattle.. $T_{A}=$ air temperature, $T_{R M}=$ mean radiant temperature, $e\left\{T_{A}\right\}=$ atmospheric partial vapour pressure, $T_{S}=$ skin temperature, $T_{B}=$ deep body temperature, $\boldsymbol{C}_{S}=$ heat flux by convection, $\boldsymbol{R}_{L}=$ radiative heat exchange (long wave), $\boldsymbol{E}_{S}=$ heat flux by cutaneous evaporation, $\boldsymbol{C}_{R}=$ sensible heat flux from the respiratory ways, $e_{S}\left\{T_{S}\right\}=$ saturation vapour pressure on the skin surface, $e_{s}\left\{T_{R}\right\}=$ saturation vapour pressure of the expired air. 
1248 Rev. bras. zootec.

$$
\mathbf{E}_{\mathrm{S}}=\frac{\rho \mathrm{c}_{\mathrm{p}}\left[e_{\mathrm{s}}\left\{\mathrm{T}_{\mathrm{S}}\right\}-e\left\{\mathrm{~T}_{\mathrm{A}}\right\}\right]}{\gamma \mathrm{r}_{\mathrm{V}}}, \mathrm{W} \cdot \mathrm{m}^{-2}
$$

e ocorre em paralelo com o fluxo de calor sensível descrito pela equação (22).

A rigor, a expressão de $\mathbf{E}_{\mathrm{S}}$ supracitada refere-se ao caso em que a superfície se acha completamente molhada e coberta por um filme de líquido, o que implicaria em taxa de sudação de, no mínimo, $1000 \mathrm{~g} \cdot \mathrm{m}^{-2} \cdot \mathrm{h}^{-1}$. Embora seja válida para eqüinos e seres humanos, no caso dos bovinos, a taxa de sudação é bem mais baixa (Tabela 1) e a superfície jamais fica molhada pelo suor produzido. Assim, é preciso incluir uma correção.

A quantidade de energia térmica necessária para converter $1 \mathrm{~g}$ de água à temperatura $\mathrm{T}_{\mathrm{S}}$ em $1 \mathrm{~g}$ de vapor à temperatura $\mathrm{T}_{\mathrm{A}}$ é dada por $\lambda-1,88\left(\mathrm{~T}_{\mathrm{S}}-\mathrm{T}_{\mathrm{A}}\right), \mathrm{J}$ conforme MONTEITH (1972), em que $\lambda$ é o calor latente de vaporização da água à temperatura $\mathrm{T}_{\mathrm{S}} \mathrm{e}$ 1,88 é o calor específico do vapor de água. Então, relacionando a quantidade de energia efetivamente dissipada com aquela que seria eliminada, se a superfície estivesse coberta com um filme de líquido, obtém-se o fator de correção:

$$
w=\frac{\gamma \mathrm{r}_{\mathrm{v}} S\left[\lambda-1,88\left(\mathrm{~T}_{\mathrm{S}}-\mathrm{T}_{\mathrm{A}}\right)\right]}{\rho \mathrm{c}_{\mathrm{p}}\left(e_{\mathrm{s}}\left\{\mathrm{T}_{\mathrm{S}}\right\}-e\left\{\mathrm{~T}_{\mathrm{A}}\right\}\right)}
$$

em que $S$ é a taxa de sudação $\left(\mathrm{g} \cdot \mathrm{m}^{-2} \cdot \mathrm{s}^{-1}\right)$.

Aplicando-se o conceito de temperatura equivalente (McARTHUR 1987), o fluxo total de calor (sensível + latente) a partir da epiderme será então:

$$
\begin{aligned}
\mathrm{Q}_{1}=\mathbf{R}_{\mathrm{L}}+\mathbf{C}_{\mathrm{S}}+\mathbf{E}_{\mathrm{S}} & =\frac{\rho \mathrm{c}_{\mathrm{p}}\left(\mathrm{T}_{\mathrm{S}}-\mathrm{T}_{\mathrm{O}}\right)}{\mathrm{r}_{\mathrm{O}}}+\frac{w \rho c_{\mathrm{p}}\left[e_{\mathrm{s}}\left\{\mathrm{T}_{\mathrm{S}}\right\}-e\right]}{\gamma \mathrm{r}_{\mathrm{V}}} \\
& =\frac{\rho c_{\mathrm{p}}}{\mathrm{r}_{0}}\left[\left(\mathrm{~T}_{\mathrm{S}}+\frac{w e_{\mathrm{s}}\left\{\mathrm{T}_{\mathrm{S}}\right\}}{\gamma_{\mathrm{s}}^{*}}\right)-\left(\mathrm{T}_{0}+\frac{w e\left\{\mathrm{~T}_{\mathrm{A}}\right\}}{\gamma_{\mathrm{s}}^{*}}\right)\right] \\
& =\frac{\rho c_{\mathrm{p}}\left(\mathrm{T}_{\mathrm{S}}^{\prime}-\mathrm{T}_{0}^{\prime}\right)}{\mathrm{r}_{0}}, \quad \mathrm{~W} \cdot \mathrm{m}^{-2}
\end{aligned}
$$

em que $\gamma_{s}^{*}=\gamma_{\mathrm{v}} / \mathrm{r}_{0}$. Em consequência, o fluxograma torna-se mais simples (Figura 2A).

Somando os fluxos $Q_{1}$ e $Q_{2}$ da Figura 2 A considerando que as resistências $r_{B}$ e $r_{0}$ estão em série, obtém-se:

$$
Q_{S}=Q_{1}+Q_{2}=\frac{\rho c_{p}\left(T_{B}-T_{S}^{\prime}\right)}{r_{B}}+\frac{\rho c_{p}\left(T_{S}^{\prime}-T_{0}^{\prime}\right)}{r_{0}}=\frac{\rho c_{p}\left(T_{B}-T_{0}^{\prime}\right)}{r_{B}+r_{0}}
$$

\begin{tabular}{|c|c|c|c|}
\hline $\begin{array}{l}\text { Raça } \\
\text { Breed }\end{array}$ & $\begin{array}{l}\text { Caso } \\
\text { Case }\end{array}$ & g. $\mathrm{m}^{-2} \cdot \mathrm{h}^{-1}$ & $\begin{array}{c}\text { Autor(es) } \\
\text { Author }(s)\end{array}$ \\
\hline $\begin{array}{l}\text { Holandesa } \\
\text { Holstein }\end{array}$ & Brasil & 275,3 & LIMA )1991) \\
\hline Jersey & Brasil & 123,3 & SILVA et al. (1988) \\
\hline Holandesa & Brasil & 185,3 & SUZUKI(1986) \\
\hline $\begin{array}{l}\text { Holstein } \\
\text { 5/8 Holandesa x Zebu } \\
\text { 5/8 Holstein x Brahman }\end{array}$ & Cuba & $264,8-327$ & MORAIS (1985) \\
\hline $\begin{array}{l}\text { 3/4 Holandesa x Zebu } \\
3 / 4 \text { Holstein } x \text { Brahman }\end{array}$ & & 263,4 & \\
\hline Holandesa & Brasil & & BORGES(1985) \\
\hline Holstein & $\begin{array}{l}\text { Verão (summer) } \\
\text { Inverno (winter) }\end{array}$ & $\begin{array}{c}249,7 \\
36,9\end{array}$ & \\
\hline $\begin{array}{l}\text { Brahman } \\
\text { Brahman x Shorthorn x Hereford } \\
\text { Shorthorn }\end{array}$ & Australia & $\begin{array}{l}294 \\
146 \\
194\end{array}$ & FINCH et al. (1982) \\
\hline Holandesa (Holstein) & Brasil, verão & 125 & SALIMOS (1980) \\
\hline Jersey & Brazil, summer & 156,1 & \\
\hline $\begin{array}{l}\text { Brahman e mestiços Europeu } \mathrm{X} \text { Zebu } \\
\text { Brahman and crossbreds }\end{array}$ & Australia & $246-500$ & SCHLEGERe TURNER(1965) \\
\hline $\begin{array}{l}\text { Holandesa } \\
\text { Holstein }\end{array}$ & $\begin{array}{c}\text { Australia, } \\
\text { Bezerros (calves) }\end{array}$ & $90-300$ & THWAITES e MOORE (1989) \\
\hline Ayrshire & $\begin{array}{c}\mathrm{T}_{\mathrm{a}}<\mathrm{TCI} \\
\mathrm{T}_{\mathrm{a}}>\mathrm{TCS}\end{array}$ & $\begin{array}{c}12-16 \\
67-144 \\
\end{array}$ & MCLEAN(1963) \\
\hline
\end{tabular}

e o fluxograma torna-se como na Figura 2B. Este fluxo está em paralelo com a transferência de calor sensível por meio da respiração $\left(\mathbf{C}_{\mathrm{R}}\right)$, cada um deles

Tabela 1 - Valores da taxa de sudação de bovinos, conforme a literatura Table 1 - Sweating rate of cattle, according to the literature 
convergindo para uma diferente temperatura ambiental. Esta transferência depende do valor da resistência $r_{H}$, a qual pode ser estimada determinando-se experimentalmente o fluxo por meio dos tecidos e aplicando-se em seguida a relação $\rho c_{p}\left(T_{B}-T_{A}\right) / r_{H}$.

$O$ valor $r_{B}$ representa a resistência dos tecidos à passagem do fluxo térmico e depende fortemente da circulação sanguínea periférica, ou seja, da vasoconstricção e da vasodilatação. A vasoconstricção ocorre quando há necessidade de limitar-se à perda de calor, nos caso em que a temperatura ambiente é baixa. A determinação de $\mathrm{rB}$ tem sido feita mais para seres humanos e animais de laboratório, havendo poucos valores publicados para bovinos. Constam da Tabela 2 algumas estimativas, sendo as fórmulas de McARTHUR (1987) provavelmente as mais indicadas para o caso presente.

Estabelecendo uma temperatura ambiental comum, $\mathrm{T}_{\mathrm{E}}$, faz-se:

$$
\frac{\rho c_{p}\left(T_{B}-T_{E}\right)}{r_{E}}=\frac{\rho c_{p}\left(T_{B}-T_{0}^{\prime}\right)}{r_{B}+r_{0}}+\frac{\rho c_{p}\left(T_{B}-T_{A}\right)}{r_{H}}
$$

sendo $r_{E}=r_{H}\left(r_{B}+r_{0}\right) /\left(r_{H}+r_{B}+r_{0}\right)$. Resolvendo para $T_{E}$, obtem-se:

$$
\mathrm{T}_{\mathrm{E}}=\frac{\mathrm{r}_{\mathrm{H}} \mathrm{T}_{0}^{\prime}+\left(\mathrm{r}_{\mathrm{B}}+\mathrm{r}_{0}\right) \mathrm{T}_{\mathrm{A}}}{\mathrm{r}_{\mathrm{H}}+\mathrm{r}_{\mathrm{B}}+\mathrm{r}_{0}}
$$

O fluxo descrito pela equação (24) acha-se em paralelo com o fluxo de calor latente a partir do sistema respiratório. Combinando ambos, obtém-se o fluxo térmico total do interior do corpo até o ambiente externo,

$\mathrm{Q}_{\mathrm{T}}=\frac{\rho \mathrm{c}_{\mathrm{p}}\left(\mathrm{T}_{\mathrm{B}}-\mathrm{T}_{\mathrm{E}}\right)}{\mathrm{r}_{\mathrm{E}}}+\frac{\rho \mathrm{c}_{\mathrm{p}}\left(e_{\mathrm{s}}\left\{\mathrm{T}_{\mathrm{R}}\right\}-e\left\{\mathrm{~T}_{\mathrm{A}}\right\}\right)}{\gamma \mathrm{r}_{\mathrm{U}}}=\frac{\rho \mathrm{c}_{\mathrm{p}}\left(\mathrm{T}_{\mathrm{BE}}-\mathrm{T}_{\mathrm{OE}}\right)}{\mathrm{r}_{\mathrm{E}}}$

em W. $\mathrm{m}^{-2}$, em que:

$$
\begin{gathered}
\mathrm{T}_{\mathrm{BE}}=\mathrm{T}_{\mathrm{B}}+\frac{\mathrm{r}_{\mathrm{E}} e_{\mathrm{s}}\left\{\mathrm{T}_{\mathrm{R}}\right\}}{\gamma \mathrm{r}_{\mathrm{U}}},{ }^{\mathrm{o}} \mathrm{K} \\
\mathrm{T}_{\mathrm{OE}}=\mathrm{T}_{\mathrm{E}}+\frac{\mathrm{r}_{\mathrm{E}} e\left\{\mathrm{~T}_{\mathrm{A}}\right\}}{\gamma_{\mathrm{U}}},{ }^{\mathrm{o}} \mathrm{K}
\end{gathered}
$$

\section{Resultados e Discussão}

$\mathrm{Na}$ equação (28), o valor $\mathrm{T}_{\mathrm{OE}}$ representa o que se pode denominar de Temperatura Operativa Equivalente do Ambiente, a qual pode ser definida como aquela combinação de fatores ambientais
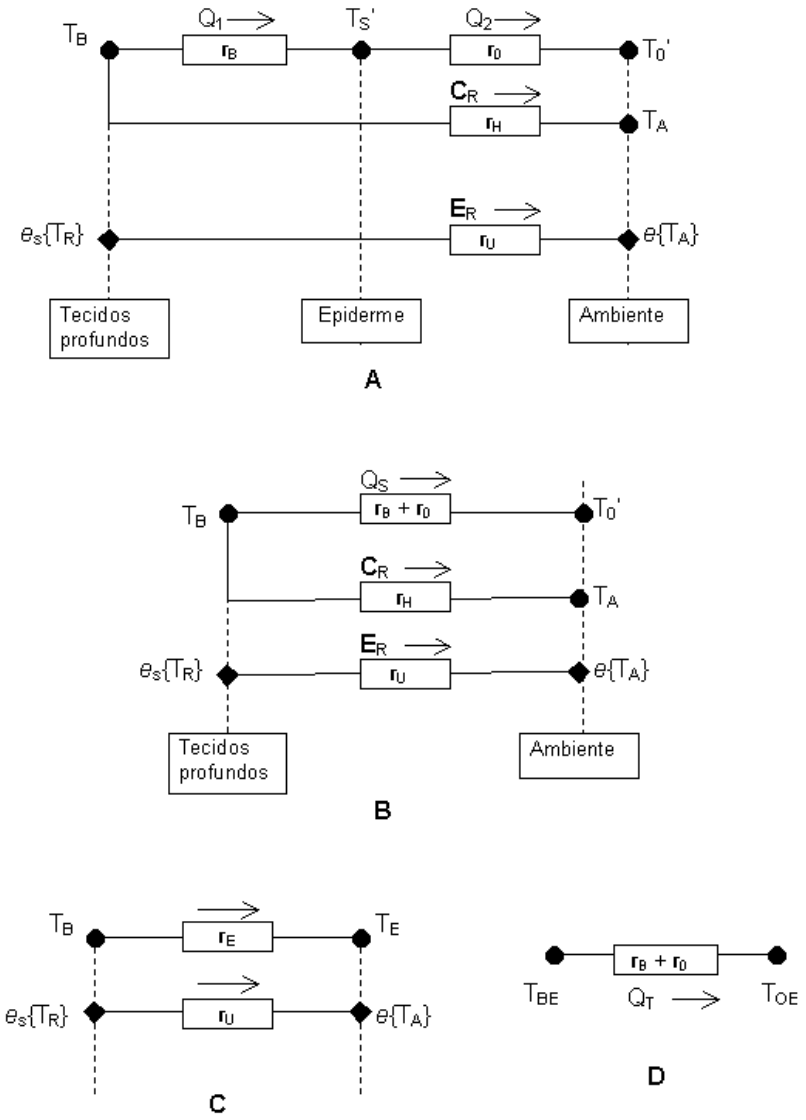

Figura 2 - Fluxos de energia térmica a partir do corpo de bovinos, em passos sucessivos de simplificação. Ver texto e legenda da Figura 1 para os símbolos.

Figure 2 - Heat flux from the body of cattle, in successive steps of simplification. For symbols, see text and the legend of Figure 1.

(temperatura radiante média, velocidade do vento, temperatura e umidade do ar), que condiciona determinado fluxo de energia térmica do interior do corpo de um animal para o ambiente externo.

Uma $\mathrm{T}_{\mathrm{OE}}$ que resulte em um valor $\mathbf{Q}_{\mathrm{T}}$ igual ao afluxo térmico $\mathbf{M}+\mathbf{R}_{\mathrm{C} \alpha}$ define um ambiente ótimo para o animal em questão, independentemente da estocagem de calor, ao passo que, quanto menor for $\mathbf{Q}_{\mathrm{T}}$ em relação a $\mathbf{M}+\mathbf{R}_{\mathrm{C} \alpha}$, maior será o estresse de calor resultante e maior a necessidade de grande capacidade de estocagem térmica.

Deve ser destacado que tal estocagem se processa enquanto a $\mathrm{T}_{\mathrm{OE}}$ está elevada durante o dia; durante a noite, mesmo em regiões tropicais, as condições ambientes permitem que o calor estocado seja rapidamente dissipado.

Na prática, em ambiente tropical, é pequena a 
1250 Rev. bras. zootec.

Tabela 2 - Resistência térmica dos tecidos em bovinos, segundo alguns autores $\left(\mathrm{t}_{\mathrm{S}}=\right.$ temperatura da superfície epidérmica)

Table 2 - Thermal resistance of peripheral tissue of cattle, according to the literature $\left(t_{S}=\right.$ surface temperature)

\begin{tabular}{|c|c|c|}
\hline $\begin{array}{l}\text { Animal/Caso } \\
\text { Animal/Case }\end{array}$ & s.m $\mathrm{m}^{-1}$ & $\begin{array}{l}\text { Autor(es) } \\
\text { Author(s) }\end{array}$ \\
\hline $\begin{array}{l}\text { Novilhos/vasoconstricção } \\
\text { Steers/vasoconstriction }\end{array}$ & 170 & Blaxter(1967) \\
\hline $\begin{array}{l}\text { Novilhos/vasodilatação } \\
\text { Steers/vasodilatation }\end{array}$ & 50 & Blaxter(1967) \\
\hline $\begin{array}{l}\text { Bezerros/vasoconstricção } \\
\text { Calves/vasoconstriction }\end{array}$ & 110 & Blaxter(1967) \\
\hline $\begin{array}{l}\text { Bezerros/vasodilatação } \\
\text { Calves/vasodilatation }\end{array}$ & 50 & Blaxter(1967) \\
\hline $\begin{array}{l}\text { Vacas } \\
\text { Cows }\end{array}$ & $12-28$ & Berman (1971) \\
\hline $\begin{array}{l}\text { Vacas Jersey } / \mathrm{t}_{\mathrm{S}}<36^{\circ} \mathrm{C} \\
\text { Jersey cows }\end{array}$ & $r_{B}=225-5,44 t_{S}$ & McArthur (1987) \\
\hline $\begin{array}{l}\text { Vacas Jersey } / \mathrm{t}_{\mathrm{S}}>36^{\circ} \mathrm{C} \\
\text { Jersey cows }\end{array}$ & 29 & McArthur (1987) \\
\hline $\begin{array}{l}\text { Bezerros Holandês x Hereford } \\
\text { Holstein } x \text { Hereford calves }\end{array}$ & $r_{B}=645-17 t_{S}$ & McArthur (1987) \\
\hline
\end{tabular}

Tabela 3 - Absorvidade da superfície externa de bovinos à radiação de ondas curtas

Table 3 - Absorptivity of haircoat surface of cattle to shortwave radiation $(0.2$ to $3.0 \mathrm{~mm})$

\begin{tabular}{|c|c|c|}
\hline $\begin{array}{l}\text { Pelame } \\
\text { Haircoat }\end{array}$ & $\alpha$ & $\begin{array}{l}\text { Autor(es) } \\
\text { Author(s) }\end{array}$ \\
\hline $\begin{array}{l}\text { Branco } \\
\text { White }\end{array}$ & $0,375-0,451 *$ & Stewart (1953) \\
\hline $\begin{array}{l}\text { Branco, assentado } \\
\text { White }\end{array}$ & 0,45 & Hutchinson e Brown (1969) \\
\hline $\begin{array}{l}\text { Branco, erecto } \\
\text { White }\end{array}$ & 0,54 & Hutchinson e Brown (1969) \\
\hline $\begin{array}{l}\text { Negro } \\
\text { Black }\end{array}$ & 0,92 & Stewart (1953) \\
\hline $\begin{array}{l}\text { Negro, assentado } \\
\text { Black }\end{array}$ & 0,91 & Hutchinson e Brown (1969) \\
\hline $\begin{array}{l}\text { Negro, erecto } \\
\text { Black }\end{array}$ & 0,92 & Hutchinson e Brown (1969) \\
\hline $\begin{array}{l}\text { Marron claro } \\
\text { Light brown }\end{array}$ & 0,675 & Stewart (1953) \\
\hline $\begin{array}{l}\text { Marron escuro } \\
\text { Dark brown }\end{array}$ & 0,725 & Stewart (1953) \\
\hline $\begin{array}{l}\text { Marron, assentado } \\
\text { Brown }\end{array}$ & 0,81 & Hutchinson e Brown (1969) \\
\hline $\begin{array}{l}\text { Vermelho escuro } \\
\text { Dark red }\end{array}$ & 0,778 & Stewart (1953) \\
\hline
\end{tabular}

* Dependendo de se achar limpo ou sujo.

* Depending on a clean or dirty surface. 
contribuição de $\mathbf{C}_{\mathrm{R}}$ para o calor total eliminado, devido ao fato de o diferencial de temperatura entre o interior do corpo e a atmosfera ser geralmente pequeno e às vezes negativo.

Em conseqüência, pode-se simplificar o processo eliminando $\mathbf{C}_{\mathrm{R}}$ e obtendo finalmente, após alguma manipulação:

$$
\begin{gathered}
\mathrm{Q}_{\mathrm{T}}=\frac{\rho \mathrm{c}_{\mathrm{p}}\left(\mathrm{T}_{\mathrm{BE}}-\mathrm{T}_{\mathrm{OE}}\right)}{\mathrm{r}_{\mathrm{B}}+\mathrm{r}_{0}}, \mathrm{~W} \cdot \mathrm{m}-2 \\
\mathrm{~T}_{\mathrm{BE}}=\mathrm{T}_{\mathrm{B}}+\frac{\mathrm{r}_{0} e_{\mathrm{s}}\left\{\mathrm{T}_{\mathrm{R}}\right\}}{\gamma \mathrm{r}_{\mathrm{V}}},{ }^{\mathrm{o}} \mathrm{K} \\
\mathrm{T}_{\mathrm{OE}}=\frac{\mathrm{r}_{\mathrm{C}} \mathrm{T}_{\mathrm{RM}}+\mathrm{r}_{\mathrm{R}} \mathrm{T}_{\mathrm{A}}}{\mathrm{r}_{\mathrm{R}}+\mathrm{r}_{\mathrm{C}}}+\frac{e\left\{\mathrm{~T}_{\mathrm{A}}\right\}}{\gamma}\left(\frac{w \mathrm{r}_{0}}{\mathrm{r}_{\mathrm{V}}}+\frac{\mathrm{r}_{\mathrm{B}}+\mathrm{r}_{0}}{\mathrm{r}_{\mathrm{U}}}\right),{ }^{\circ} \mathrm{K}
\end{gathered}
$$

A partir das equações acima, é possível estimar o nível de produção de calor metabólico que pode equilibrar a termólise nas condições ambientais proporcionadas pela temperatura operativa equivalente e pela radiação solar de ondas curtas:

$$
\mathbf{M}=\frac{\rho \mathrm{c}_{\mathrm{p}}\left(\mathrm{T}_{\mathrm{BE}}-\mathrm{T}_{\mathrm{OE}}\right)}{\mathrm{r}_{\mathrm{B}}+\mathrm{r}_{0}}-\mathbf{R}_{\mathrm{C}} \alpha
$$

em que $T_{B E}$ e $T_{O E}$ são dadas pelas equações [30] e [31], respectivamente. Se o valor assim estimado for muito menor que o nível real de metabolismo, $\mathbf{M}^{*}$ — por exemplo, quando há alta produção dos animais - então a diferença de energia deverá ser estocada, $\mathbf{S}=\mathbf{M}^{*}$ - $\mathbf{M}$, até um limite que não pode ser ultrapassado. Esse problema é um indício de que (a) há necessidade de alteração do ambiente em que se acham os animais, inclusive manejo, de modo a reduzir a exposição à radiação e favorecer os mecanismos termolíticos; (b) as características dos animais são inadequadas para esse ambiente.

\section{Aplicação}

Seja uma vaca Holandesa de $350 \mathrm{~kg}$, diâmetro corporal médio $0,9 \mathrm{~m}$, com pêlos curtos e bem assentados, predominantemente negra, colocada à sombra de um abrigo onde não há radiação solar direta, sendo a radiação difusa de ondas curtas $\mathbf{R}_{\mathrm{c}}=100 \mathrm{~W} \cdot \mathrm{m}^{-2}$, a temperatura de bulbo seco de $32^{\circ} \mathrm{C}$, a temperatura de bulbo úmido de $29^{\circ} \mathrm{C}$, a velocidade do vento de $1,5 \mathrm{~m} . \mathrm{s}^{-1} \mathrm{e}$ a temperatura de globo de $35^{\circ} \mathrm{C}$. A temperatura retal é $39,9^{\circ} \mathrm{C}$; a da superfície cutânea, $36^{\circ} \mathrm{C}$; a frequência respiratória, $100 \mathrm{~min}^{-1}$; e a taxa de sudação, $275 \mathrm{~g} \cdot \mathrm{m}^{-2} \cdot \mathrm{h}^{-1}$.

A temperatura da superfície corporal foi determinada com um radiômetro de infravermelho Horiba IT-330 e a temperatura radiante média, calculada a partir da temperatura de globo, usando as fórmulas de SILVA (1999), obtendo-se, respectivamente, $\mathrm{T}_{\mathrm{S}}=309,15^{\circ} \mathrm{Ke} \mathrm{T}_{\mathrm{RM}}=309,6^{\circ} \mathrm{K}$. As características do ar a $32^{\circ} \mathrm{C}$ são: $\rho=1,158 ; \lambda=2425,1 ; \gamma=0,0666$; $\mathrm{k}=0,0265 ; \mathrm{v}=1,617 \times 10^{-5} ; D=2,599 \times 10^{-5} \mathrm{e}$ $c_{p}=1006,5$. Aplicando as fórmulas dadas, os demais valores calculados são:

$$
\begin{array}{lll}
\mathrm{R}_{\mathrm{e}}=83.487,9 & \mathrm{r}_{\mathrm{C}}=192,46 \mathrm{~s} \cdot \mathrm{m}^{-1} \\
\mathrm{P}_{\mathrm{r}}=0,71119 & \mathrm{r}_{\mathrm{V}}=176,91 \mathrm{~s} \cdot \mathrm{m}^{-1} \\
\mathrm{~N}_{\mathrm{u}}=205,68 & \mathrm{r}_{\mathrm{U}}=3125 \mathrm{~s} \cdot \mathrm{m}-1 \\
\mathrm{~S}_{\mathrm{h}}=195,75 & \mathrm{r}_{\mathrm{R}}=176,72 \mathrm{~s} \cdot \mathrm{m}-1 \\
\mathrm{~S}=0,076389 \mathrm{~g} \cdot \mathrm{m}^{-2} \cdot \mathrm{s}^{-1} & \mathrm{r}_{0}=92,125 \mathrm{~s} \cdot \mathrm{m}^{-1} \\
w \quad=0,876 & \mathrm{r}_{\mathrm{B}}=29,16 \mathrm{~s} \cdot \mathrm{m}^{-1} \\
\mathrm{~T}_{\mathrm{OE}}=335,7^{\circ} \mathrm{K} & \mathrm{T}_{\mathrm{BE}}=367,9^{\circ} \mathrm{K} \\
& \mathrm{Q}_{\mathrm{T}}=309,4 \mathrm{~W} \cdot \mathrm{m}^{-2}
\end{array}
$$

$$
\mathbf{M}=\mathbf{Q}_{\mathrm{T}}-\mathbf{R}_{\mathrm{C} \alpha}=309,4-(100)(0,92)=217,4 \mathrm{~W} \cdot \mathrm{m}^{-2} .
$$

Portanto, para equilibrar a termólise nas condições indicadas, o animal deve apresentar uma termogênese em nível de 217,4 W.m². A taxa metabólica mínima estimada pela equação [2a] é 81,5 $\mathrm{W} . \mathrm{m}^{-2}$, de modo que nessas condições o animal não apresentará qualquer problema de estresse térmico. A diferença $217,4-81,5=135,9 \mathrm{~W} . \mathrm{m}^{-2}$ inclui o calor gerado pela produção e por outras fontes.

\section{Referências bibliográficas}

BERMAN, A. 1971. Thermoregulation in intensively lactating cows in near-natural conditions. J. Physiol., 215:477-482.

BLAXTER, K. 1967. The energy metabolism of ruminants. 2.ed. London: Hutchinson. 250p.

BLAXTER, K. 1989. Energy metabolism in animals and man. Cambridge: Cambridge Univ. Press. 336p.

BORGES, F.M.A. Relações entre as caraterísticas do pelame, a taxa de sudação e o comportamento de vacas Holandesas na pastagem. Jaboticabal: FCAVJ-UNESP, 1985. 40p. Monografia (Graduação em Zootecnia) - Universidade Estadual Paulista, 1985.

CHAPMAN, A.J. 1987. Fundamentals of heat transfer. New York: MacMillan. 751p.

FINCH, V.A. 1985. Comparison of non-evaporative heat transfer in different catle breeds. Aust. J. Agric. Res., 36:497-508.

FINCH, V.A., BENNETT, I.L., HOLMES, C.R. 1982. Sweating response in cattle and its relation to rectal temperature, tolerance of sun and metabolic rate. J. Agric. Sci., 99:479-487.

HERRINGTON, L.P., WINSLOW, C.E.A., GAGGE, A.P. 1937. The relative influence of radiation and convection upon vasomotor temperature regulation. Amer. J. Physiol., 120:133-143.

HUTCHINSON, J.C.D., BROWN, G.D. 1969. Penetrance of cattle coats by radiation. J. Applied Physiol., 26:454-464.

KIBLER, H.H., BRODY, S. 1950. Environmental Physiology XI. Effects of temperature, $50^{\circ}$ to $150^{\circ} \mathrm{F}$ on heat production and cardiorespiratory activities in Brahman, Jersey, and Holstein cows. Missouri Agr. Exp. Sta. Res. Bull., n. 461, 29p.

KLEIBER, M. 1961. The fire of life. New York: J. Wiley. 454p.

LIMA, L.O.S.L. Aspectos genéticos da relação entre a taxa de sudação e a produção de leite em vacas da raça Holandesa. 
1252 Rev. bras. zootec.

Jaboticabal, SP: FCAVJ, 1991. 62p. Tese (Doutorado em Zootecnia), Faculdade de Ciências Agrárias e Veterinárias/ Universidade Estadual Paulista, 1991.

McARTHUR, A.J. 1987. Thermal interaction between animal and microclimate: a comprehensive model. J. Theoret. Biol., 126:203-238.

McLEAN, J.A. 1963. The regional distribution of cutaneous moisture vaporization in the Ayrshire calf. J. Agric. Sci., 61:275-280.

McLEAN, J.A., DOWNIE, A.J., JONES, C.D.R. et al. 1983a. Thermal adjustments of steers (Bos taurus) to abrupt changes in environmental temperature. J. Agric. Sci., 100:305-314.

McLEAN, J.A., STOMBAUGH, D.P., DOWNIE, A.J. et al. 1983b. Body heat storage in steers (Bos taurus) in fluctuating thermal environments. J. Agric. Sci., 100:315-322.

MONTEITH, J.L. 1972. Latent heat of vaporization in thermal physiology. Nature News Biol., 236:96.

MONTEITH, J.L. 1974. Specification of the environment for thermal physiology. In: MONTEITH, J.L., MOUNT, L.E. (Eds.) Heat loss from animals and man, London: Butherworths. p.1-17.

MONTEITH, J.L., UNSWORTH, M.H. 1991. Principles of environmental physics. 2.ed. London: E. Arnold. 291p.

MORAIS, M. 1985. Tolerancia al calor de bovinos HolsteinCebu. II. Efecto de la temperatura ambiental y la humedad relativa en la temperatura rectal, frecuencia respiratoria y tasa sudoral en novillos cruzados. Rev. Cubana Ciencias Vet., 16:193-200.

OWEN, E., KAVLE, A., OWEN, R.S. et al. 1986.A reappraisal of caloric requirements in healthy woman. Amer. J. Clin. Nut., 44:1-19.

PORTER, W.P., GATES, D.M. 1969. Thermodynamic equilibria of animals with environment. Ecol. Monogr., 39:227-235.

SALIMOS, E.P. Alguns fatores que afetam a função sudorípara em vacas Jersey e Holandesas. Jaboticabal: FCAVJ-UNESP, 1980. 44p. Dissertação (Mestrado em Zootecnia) - Universidade Estadual Paulista, 1980.

SCHLEGER, A.V., TURNER, H.G. 1965. Sweating rates of cattle in the field and their reaction to diurnal and seasonal changes. Aust. J. Agric. Res., 16:92-106.

SCHMIDT-NIELSEN, K. 1989. Scaling: why is animal size so important? Cambridge: Cambridge Univ. Press. 241p.

SILVA, R.G. 1999a. Estimativa do balanço térmico por radiação em vacas Holandesas expostas ao sol e à sombra em ambiente tropical. Rev. bras. zootec., 28(6):1403-1411.

SILVA, R.G. 1999b. Introdução à bioclimatologia animal. São Paulo: Nobel. 415p.

SILVA, R.G., ARANTES NETO, J.G., HOLTZ FILHO, S.V. 1988. Genetic aspects of the variation of the sweating rate and coat characteristics of Jersey cattle. Braz. J. Genet. 11:335-347.

STEVENS, D.G. 1981. A model of respiratory vapor loss in Holstein dairy cattle. Transactions of the ASAE, 24:151-158.

STEWART, R.E. 1953. Absorption of solar radiation by the hair of cattle. Agric.Engineering, 34:235-238.

SUZUKI, A.H. Sudação e produção de leite em vacas Holandesas em clima tropical. Jaboticabal: FCAVJ-UNESP, 1986. 21p. Monografia (Graduação em Zootecnia) - Universidade Estadual Paulista, 1986.

THWAITES, C.J., MOORE, I.S.F. 1989. Development of sweating ability in winter- and summer-born Friesian calves aged 1 to 6 weeks. Int. J. Biometeorol., 33:246-250.

\section{Apêndice}

Fórmulas para obtenção de características do ar no intervalo de temperaturas $\left(t_{A}\right)$ entre -5 e $50^{\circ} \mathrm{C}$ :

\section{Viscosidade cinemática}

$v=1,32909 \times 10^{-5}+9 \times 10^{-8} \mathrm{t}_{\mathrm{A}}, \mathrm{m}^{2} \cdot \mathrm{s}^{-1}$

\section{Constante psicrométrica}

$\gamma=.0645818+6.3636 \times 10^{-5} \mathrm{t}_{\mathrm{A}}, \mathrm{kPa} \cdot{ }^{\circ} \mathrm{C}^{-1}$

\section{Densidade}

$\rho=1.289764-.004111 \mathrm{t}_{\mathrm{A}}, \quad \mathrm{kg} \cdot \mathrm{m}^{-3}$

\section{Condutividade térmica}

$\mathrm{k}=.024324+6.2909 \times 10^{-5} \mathrm{t}_{\mathrm{A}}, \mathrm{W} \cdot \mathrm{m}^{-1} \cdot{ }^{\circ} \mathrm{C}^{-1}$

Difusividade do vapor de água

$D=2.12109 \times 10^{-5}+1.494548 \times 10^{-7} \mathrm{t}_{\mathrm{A}}, \mathrm{m}^{2} \cdot \mathrm{s}^{-1}$

\section{Calor específico}

$c_{p}=1005.524+.033714 t_{A}, J \cdot \mathrm{kg}^{-1} \cdot{ }^{\circ} \mathrm{C}^{-1}$

\section{Calor latente de vaporização da água}

$1=2500.7879-2.37374 t_{A}, J g^{-1}$

Fórmula para estimação da aceleração da gravidade conforme a latitude L (graus) e a altitude (metros):

$g=9,7743+0,00527 \mathrm{e}^{\mathrm{L} / 23,7842}-0,0031 \mathrm{~h} / 1000, \mathrm{~m} \cdot \mathrm{s}^{2}$

Recebido em: 03/08/99

Aceito em: 09/11/99 